\section{The effects of experimenter expectancy on guessing performance and level of confidence in guessing accuracy}

\author{
VICTOR R. MARTUZA \\ University of Delaware, Newark, Del. 19711
}

\begin{abstract}
An exploratory study was conducted to examine the effects of $E$ expectancy on guess-type data and to find out if $S$ 's level of confidence in the correctness of each guess was related to the consistency of his guessing performance with that expected by $\mathrm{E}$. To accomplish this, $15 \mathrm{Es}$ and 64 Ss were assigned randomly to three experimental conditions, differing only in the information given $\mathbf{E}$ about the number of "head" responses his Ss typically emitted in a sequence of coin toss guesses. The task required each $S$ to guess the outcomes of 30 consecutive coin tosses and to express his level of confidence, on a 10-point scale, in the correctness of each guess. The $E$ expectancy effect on the coin toss data was nonsignifjcant, but analysis of the confidence data suggested that S's level of confidence in the correctness of his guesses might be related to the consistency between E's expectation regarding S's guessing performance and the data he actually receives from $S$.
\end{abstract}

During the past decade, considerable interest has been shown in the $\mathbf{E}$ expectancy effect-i.e., the effect of E's research hypothesis on the data he collects. Although E effects have been demonstrated, using a variety of tasks (see Rosenthal, 1969), a large proportion of the expectancy studies have involved the standard person-perception procedure developed by Rosenthal \& Fode (1963). To explore the task generality of this phenomenon further, the present experiment required each $S$ to guess the outcome of a series of coin toss events and to express his level of confidence in the correctness of each guess. The latter task was included to detect any secondary bias effects which might result from an incongruity between E's expectation and the data he collects in the coin toss task. That such an effect is possible has been suggested by the results obtained in several studies. For example, Rosenthal, Persinger, Vikan-Kline, \& Fode (1963) and Rosenthal, Kohn, Greenfield, \& Carota (1965) used accomplices to manipulate the degree of congruity between $E$ 's expectations about $S$ performance and the early returns $E$ received from his Ss. In both studies, Es receiving confirmatory data in the early portion of the experiment tended to receive late returns which were also consistent with their expectations. As a result of these findings, Rosenthal (1966, p. 203) conjectured that the reception of disconfirming data by $E$ might result in E's becoming less "likable," "personal," and "interested" and, therefore, less effective as an expectancy-biasing $E$. The principal limitation of these results seems to be their restriction to the person-perception tasks from which they derive.

The purpose of the present study was the demonstration of the $E$ bias phenomenon, utilizing two novel tasks: (1) a series of "guessed" coin toss outcomes and (2) an expression of confidence in each "guessed" outcome. The two hypotheses tested are: (1) different "head" outcome expectancies will affect differentially the number of "head" responses obtained, and (2) Es receiving coin toss data consistent with their expectations will obtain mean confidence scores from their Ss that are different from those of Es receiving coin toss data inconsistent with their expectations.

\section{ASSISTANTS}

Three male and two female graduate students attending the College of Education at the University of Maryland voluntarily served as research assistants. Their approximate age range was 20-30 years.

\section{EXPERIMENTERS}

Six male and nine female graduate education students enrolled in an introductory research course approximate age range was $20-40$ years.

Fourteen male and 50 female graduate students enrolled in an introductory educational measurement course voluntarily served as Ss. Their approximate age range was. 20.40 years. Participation in the study was during released class time.

\section{APPARATUS}

The apparatus consisted of black $4 \times 6$ in. binders, containing 31 blue $3 \times 5$ in. cards, a $12 \times 18$ in. Masonite voluntarily served as the Es. Their shield, a stopwatch, specially prepared I BM response forms, and $S$ information cards. The first card in the stimulus presentation device (binder) was blank. Of the remaining cards, 15 displayed the letter " $H$ " and 15 , the letter "T." The randomly determined order was T T H T T TH T TH TH H H T H T T TH TH TH TH TH. Each subject information card displayed $S$ 's name, ID number, sex, fictitious "pretest" score, and bias group membership. The latter appeared as a handwritten comment in the lower right corner of the card.

Task

The experimental task consisted of 30 trials. On each trial, $S$ was required to guess the outcome of one hypothetical coin toss (head or tail) and to express his confidence in the guess on an equal unit scale ranging from extreme lack of confidence $(0)$ to very high degree of confidence (9). Ten seconds were allowed for each response. All responses were recorded by $E$. Upon the completion of each trial, feedback concerning the outcome of the coin toss was given to S.

\section{Procedure}

Three weeks before the experimental session, the $S$ s were asked to complete a task designed to obtain normative data for a "guessing-behavior" study. The task consisted of the construction of a random sequence of 30 , coin toss outcomes and the most likely answer pattern for a 20-item, 4-option, multiple-choice exam. A number of characteristics observed in the coin toss data were subsequently utilized in establishing the "guessing-behavior" rationale for the present study.

Two weeks later, the Es were trained in the mechanical aspects of experimental execution. Each $\mathbf{E}$ was allowed to play the role of both $\mathrm{E}$ and $S$ in separate trial runs.

For the experiment, all Ss and Es were assigned randomly to one of three treatment or expectancy groups: (1) the low-heads group (10-13 head-response expectancy), (2) the neutral group (14-16 head-response expectancy), or (3) the high-heads group (17-20 head-response expectancy). The Ss were also assigned a fictitious "pretest" score by a random process in the ranges shown above. Each E ran 3-5 Ss. The treatment group sizes were 22,23 , and 19 , respectively.

The assistants (As) met with the author twice prior to data collection and were thoroughly instructed in their role. As were assigned randomly to treatment groups.

At execution time, $S$ was escorted to a predetermined experimental area where he was greeted and detained 
Table 1

Mean Number of "Heads" Per Block of Five Responses for Three Treatment Groups

\begin{tabular}{cccccccc}
\hline & \multicolumn{6}{c}{ Block } & \\
\cline { 2 - 7 } Group & 1 & 2 & 3 & 4 & 5 & 6 & \\
\hline 1 & 3.45 & 2.50 & 2.59 & 2.18 & 2.27 & 2.59 & 2.60 \\
2 & 3.48 & 1.87 & 2.48 & 2.35 & 2.78 & 2.35 & 2.55 \\
3 & 3.58 & 2.37 & 2.68 & 2.42 & 3.00 & 2.47 & 2.73 \\
& 3.50 & 2.25 & 2.58 & 2.32 & 2.68 & 2.47 & 2.64 \\
\hline
\end{tabular}

Table 2

Mean Confidence Level Per $S$ Per Block of Five Responses for Three Treatment Groups

Block

\begin{tabular}{cccccccc}
\cline { 2 - 6 } Group & 1 & $\mathbf{2}$ & $\mathbf{3}$ & $\mathbf{4}$ & $\mathbf{5}$ & $\mathbf{6}$ & \\
\hline 1 & 5.27 & 5.35 & $\mathbf{5 . 2 5}$ & $\mathbf{5 . 1 0}$ & $\mathbf{5 . 0 9}$ & $\mathbf{4 . 6 8}$ & $\mathbf{5 . 1 2}$ \\
2 & $\mathbf{5 . 7 7}$ & $\mathbf{5 . 3 8}$ & $\mathbf{5 . 7 7}$ & $\mathbf{5 . 6 3}$ & $\mathbf{5 . 2 6}$ & $\mathbf{5 . 6 8}$ & $\mathbf{5 . 5 8}$ \\
$\mathbf{3}$ & $\mathbf{6 . 0 7}$ & $\mathbf{5 . 3 9}$ & $\mathbf{5 . 4 8}$ & $\mathbf{5 . 6 8}$ & $\mathbf{5 . 2 8}$ & $\mathbf{5 . 6 4}$ & $\mathbf{5 . 5 9}$ \\
& $\mathbf{5 . 7 0}$ & $\mathbf{5 . 3 7}$ & $\mathbf{5 . 5 0}$ & $\mathbf{5 . 4 7}$ & $\mathbf{5 . 2 1}$ & $\mathbf{5 . 3 3}$ & $\mathbf{5 . 4 3}$ \\
\hline
\end{tabular}

momentarily by $A$. The $S$ information card was then given to $E$ by $A$. $E$ was reminded to record the information from that card on an IBM response fo $\cdot \mathrm{m}$. In addition, $\mathrm{E}$ was asked to record S's group membership (high-heads group, neutral group, or low-heads group) at the top center of the response form. This act, coupled with the recording of S's "pretest" score in the ID block of the response form, constituted the expectancy inducement. $S$ was then instructed to enter the experimental area, and $A$ left. $E$ read the instructions to $S$ and then presented the experimental task. The response form and $S$ information card were not visible to $S$ at any time.

From the outset, all participants were urged to avoid discussing the experiment. Questions asked by Es and Ss during the course of the experiment indicated a belief that guessing behavior was the variable under investigation. There was no evidence to the contrary.

ANALYSIS AND DISCUSSION

The trials were grouped in six blocks of five consecutive trials each, and the group by block cell means were computed for both the coin toss and confidence data (see Tables 1 and $2)$. These cell means were then analyzed as a one-factor repeated measures design. While this is perhaps an overly conservative procedure, it seems justified in view of the preliminary nature of this study.

Analysis of the coin toss data did not reveal a significant difference in the number of "head" responses $(\mathrm{F}=1.48, \mathrm{df}=2,10, \mathrm{p}>.05)$ obtained by differentially biased Es (see Table 1). Several explanations of this result seem plausible.

First, some of the Es may have been too preoccupied with the mechanics of execution to mediate effectively or even to possess a strong outcome bias. Friedman, Kurland, \& Rosenthal (1965) found that the molar E characteristics of "dominance" and "professional" tend to correlate highly with the magnitude of bias transmitted to $\mathrm{S}$. It seems reasonable to postulate that $S$ may perceive a less dominant or less professional $\mathbf{E}$ when the latter displays concern over trivial details, procedural uncertainty, or nervousness during the experiment.

Secondly, the tendency to respond, "heads," may be too strong to be affected appreciably by an E's expectancy or bias. A more nearly neutral set of stimuli (i.e., geometric figures; nonsense syllables, etc.) may provide a solution to this problem, if in fact, this is the reason for the present result.

Finally, the effect of feedback on subsequent responses may tend to dilute or negate the effects of expectancy transmission to the student. Replication of the experiment without the provision of explicit feedback may yield quite different results and seems worth pursuing.

Analysis of the confidence data, on the other hand, revealed a significant "treatment" effect $(F=8.97$, $\mathrm{df}=2,10, \quad \mathrm{p}<.01)$. Using Scheffé's procedure, mean confidence level of the low-heads group was found to be significantly lower $(p<.01)$ than the average confidence level obtained from the other two groups. Examination of the coin-toss data in Table 1 shows that the low-heads group received coin toss responses inconsistent with their expectations, while the data collected by the other two groups of Es seem quite compatible with their expectations, especially in Block 1. Further inspection of the confidence data of the low-heads group reveals a weak downward trend across Blocks 2-5, with a precipitous drop occurring in the final block. On the other hand, the cell means obtained from the other groups are consistently higher than those obtained from the low-heads group and, except for Block 5, seem to suggest negatively accelerated confidence curves.

These results suggest the possibility that the degree of congruence (or incongruence) between E's expectation regarding $S$ 's performance and the data he actually receives from $S$ may, in some way, alter E's behavior toward $S$, resulting in a progressive modification of S's level of confidence as the process of data collection procedes. If this is so, then a question arises concerning the possibility of a residual effect on an individual's level of confidence when he responds to an interviewer, teacher, or counselor in a manner inconsistent with the latter's expectation regarding his performance. Of course, this interpretation is highly speculative, and further research is necessary before any firm conclusions can be drawn concerning the connection between $E^{\prime}$ s expectancy/data congruence and S's level of confidence concerning the accuracy (or perhaps even appropriateness) of his behavior in the presence of $\mathrm{E}$.

In addition, the findings of the present study suggest that guessing behavior may be less susceptible than confidence to $E$ 's influence. However, minor modifications of the coin task, as suggested earlier, may offer a means for also demonstrating this phenomenon on "guessing" performance. If this fails, a shift of focus to the direct biasing of the expressed confidence level may yield a sufficiently stable result to permit the study of the effect of other variables on the $\mathrm{E}$ bias phenomenon itself.

\section{REFERENCES}

FRIEDMAN, N., KURLAND, D., \& ROSENTHAL, R. Experimenter behavior as an unintended determinant of experimental results. Joumal of Projective Techniques \& Personality Assessment, 1965, 29, 479-490.

ROSENTHAL, R. Experimenter effects in behavioral research. New York: Appleton-Century-Crofts, 1966.

ROSENTHAL, $R$. Task variations in studies of experimenter expectancy effects. Perceptual \& Motor Skills, 1969, 29, 9-10.

ROSENTHAL, R., \& FODE, K. L. Psychology of the scientist: $V$. Three experiments in experimenter bias. Psychological Reports, 1963, 12, 491-511.

ROSEN TH A L , R , KOH N, R , GREENFIELD, P. M., \& CAROTA, N. Psychology of the scientist: XIV. Experimenters' hypothesis-confirmation and mood as determinants of experimental results. Perceptual \& Motor Skills, 1965, 20, 1237-1252.

ROSENTHAL, R., PERSINGER, G. W., VIKAN-KLINE, L., \& FODE, $K$. L. The effect of early data returns on data subsequently obtained by outcome-biased experiments. Sociometry, 1963,26 , 487-498. 